\title{
The PLATO observatory: robotic astronomy from the Antarctic plateau
}

M. C. B. Ashley ${ }^{1}$, G. Allen ${ }^{2}$, C. S. Bonner ${ }^{1}$, S. G. Bradley ${ }^{3}$, X. Cui ${ }^{4}$, J. R. Everett ${ }^{1}$, L. Feng ${ }^{5}$, X. Gong ${ }^{4}$, S. Hengst ${ }^{1}$, J. Hu ${ }^{6}$, Z. Jiang ${ }^{6}$, C. A. Kulesa ${ }^{7}$, J. S. Lawrence ${ }^{1,8,9}$, Y. Li ${ }^{10}$, D. M. Luong-Van ${ }^{1}$, M. J. McCaughrean ${ }^{11,12}$, A. M. Moore ${ }^{13}$, C. Pennypacker ${ }^{14}$, W. Qin ${ }^{10}$, R. Riddle ${ }^{13}$, Z. Shang ${ }^{16}$, J. W. V. Storey ${ }^{1}$, B. Sun ${ }^{10}$, N. Suntzeff ${ }^{17}$, N. F. H. Tothill ${ }^{11}$, T. Travouillon ${ }^{15}$, C. K. Walker ${ }^{7}$, L. Wang ${ }^{5,17}$,

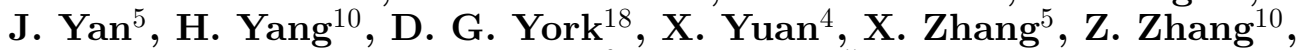
$\mathrm{X} \cdot \mathrm{Zhou}^{6}$ and $\mathrm{Z} \cdot \mathrm{Zhu}^{5}$

${ }^{1}$ School of Physics, University of New South Wales, NSW 2052, Australia

${ }^{2}$ Solar Mobility Pty Ltd, Thornleigh, NSW 2120, Australia

${ }^{3}$ Physics Department, University of Auckland, Auckland 1142, New Zealand

${ }^{4}$ Nanjing Institute of Astronomical Optics and Technology, Nanjing 210042, China

${ }^{5}$ Purple Mountain Observatory, Nanjing 210008, China

${ }^{6}$ National Astronomical Observatories, Chinese Academy of Sciences, Beijing 100012, China

${ }^{7}$ Steward Observatory, University of Arizona, Tucson, AZ 85721, USA

${ }^{8}$ Department of Physics and Engineering, Macquarie University, NSW 2109, Australia

${ }^{9}$ Anglo-Australian Observatory, NSW 1710, Australia

${ }^{10}$ Polar Research Institute of China, Pudong, Shanghai 200136, China

${ }^{11}$ School of Physics, University of Exeter, Exeter, EX4 4QL, UK

${ }^{12}$ European Space Agency, ESTEC, Postbus 299, 2200 AG Noordwijk, The Netherlands

${ }^{13}$ Caltech Optical Observatories, California Institute of Technology, Pasadena, CA 91125, USA

${ }^{14}$ Lawrence Berkeley National Laboratory and Space Sciences Laboratory, University of California, Berkeley, CA 94720, USA

${ }^{15}$ Thirty Meter Telescope Project, Pasadena, CA 91107, USA

${ }^{16}$ Tianjin Normal University, Tianjin 300074, China

${ }^{17}$ Physics Department, Texas A\&M University, College Station, TX 77843, USA

${ }^{18}$ Department of Astronomy and Astrophysics and The Enrico Fermi Institute, University of Chicago, Chicago, IL 60637, USA

\begin{abstract}
PLATO is a 6 tonne completely self-contained robotic observatory that provides its own heat, electricity, and satellite communications. It was deployed to Dome A in Antarctica in January 2008 by the Chinese expedition team, and is now in its second year of operation. PLATO is operating four $14.5 \mathrm{~cm}$ optical telescopes with $1 \mathrm{k} \times 1 \mathrm{k}$ CCDs, a wide-field sky camera with a $2 \mathrm{k} \times 2 \mathrm{k}$ CCD and Sloan g, r, i filters, a fibre-fed spectrograph to measure the UV to near-IR sky spectrum, a $0.2 \mathrm{~m}$ terahertz telescope, two sonic radars giving $1 \mathrm{~m}$ resolution data on the boundary layer to a height of $180 \mathrm{~m}$, a $15 \mathrm{~m}$ tower, meteorological sensors, and 8 web cameras. Beginning in 2010/11 PLATO will be upgraded to support a Multi Aperture Scintillation Sensor and three AST3 $0.5 \mathrm{~m}$ schmidt telescopes, with $10 \mathrm{k} \times 10 \mathrm{k}$ CCDs and $100 \mathrm{~TB} /$ annum data requirements.
\end{abstract}

Keywords. Site Testing, Instrumentation: miscellaneous, Atmospheric Effects, Telescopes 


\section{Introduction}

The potential of Antarctica, in particular the high plateau in the Antarctic interior, to provide the best astronomical observing sites on the Earth's surface has been widely discussed in the literature (see, e.g., Ashley et al. 2004). Dome C (3260m altitude) on the plateau shows a median seeing of 0.27 arcseconds above a $30 \mathrm{~m}$ turbulent boundary layer (Lawrence et al. 2004). However, some of the best potential sites, such as Dome A, do not yet have the infrastructure to support people over the winter. To explore these sites, both for site-testing and for simple astronomical experiments, it is necessary to have a reliable source of power and internet connectivity. Hence PLATO.

PLATO, short for "plateau observatory", is a self-contained astronomical observatory designed to provide $1 \mathrm{~kW}$ of electricity in order to run experiments with no human presence for up to a year before servicing (Lawrence et al. 2009). Electricity is generated by a combination of solar power and diesel engines running on Jet-A1 fuel. PLATO has "on-board" supervisor computers that provide internet access via Iridium modems, and allow many aspects of the facility to be controlled. For the convenience of instrument designers PLATO provides a thermally-insulated environment inside a 10-foot shipping container that can be temperature controlled, usually at least $50^{\circ} \mathrm{C}$ above the ambient temperature - which can fall to below $-75^{\circ} \mathrm{C}$ in winter.

\section{PLATO Performance}

\subsection{Power system}

PLATO was installed at Dome A by the Chinese expedition team during January 2008, and ran for 204 days that year, stopping due to an exhaust leak from its engines in early August. At the time of writing (2009 November 9), PLATO has been running for 301 days continuously. Details of the engine system are given by Hengst et al. 2009 .

\subsection{Iridium communications}

With two Iridium modems, PLATO can reliably transfer $\sim 30 \mathrm{MB}$ of data per day from Antarctica. The transfer occurs over an "ssh" socket connection, and uses a custom Perl script that copes efficiently with the partial transfer of large files, while simultaneously allowing bidirectional control of PLATO via a "bash" command-line interface. Iridium also provides reliable absolute time for the PLATO instruments, accurate to $\pm 20 \mathrm{~ms}$.

\subsection{Scientific instruments}

The original PLATO instruments, and results from 2008, are described by Yang et al. 2009. The PreHEAT instrument showed spectacularly-high atmospheric transmission at a wavelength of 450 microns - a paper describing these results is in preparation.

During 2009, the instruments described in the abstract have been operating. All have worked well and returned data for much of the year. Three papers on CSTAR results are in preparation. Snodar (Bonner et al. 2009) has given excellent statistical information on the height of the atmospheric boundary layer, with 10 second or better time resolution, and $1 \mathrm{~m}$ spatial resolution, throughout the year.

\subsection{Diagnostic information}

The data stream from PLATO includes health and status information such as bus voltages and engine temperatures. This information is available from a webpage updated every minute, with the data usually between 1 and 4 minutes old. Any anomalies with the data trigger the transmission of an SMS message to one or more mobile phones. In practice, 
PLATO can operate for weeks at a time with no need for outside intervention. Such intervention is usually only necessary to change instrument parameters or to work around sub-system failures. The redundant nature of much of PLATO's design has allowed us to continue operating despite the occasional electrical and mechanical problems.

There are 8 web-cameras of various types to monitor the sky conditions and instrument icing. One of the cameras is inside the Engine Module, and can assist with diagnosing engine problems such as the exhaust leak that stopped PLATO during 2008. This camera includes a microphone, to measure the engine RPM and general health.

\section{Future Plans}

PLATO is serviced each Austral summer by the Chinese expedition team organized by the Polar Research Institute of China. At a minimum, servicing involves replacing the six diesel engines, changing the lubricating oil, and filling the Jet-A1 fuel tank. The opportunity is also taken to maintain and upgrade the scientific experiments.

For 2010 we are adding a sub-millimeter Fourier Transform Spectrometer, and a lunar SHABAR to measure the contribution of the boundary layer to astronomical seeing.

Beyond 2010, we will need to replace PLATO's Engine Module with a higher-power version to support three 0.5-m Antarctic Schmidt Telescopes (AST3) under construction at NIAOT. There are also plans to install a Multi Aperture Scintillation Sensor to provide data on the free atmosphere contribution to the astronomical seeing.

\section{Conclusions}

The PLATO concept has proven its reliability through two successful periods of operation during 2008 and 2009. In practice, the lack of on-site people during winter has not been a major impediment, and has had some benefits: e.g., the instruments have to be designed from the outset for full automation, which tends to lead to greater reliability and longer uptimes.

\section{Acknowledgements}

We thank the Polar Research Institute of China for making our productive collaboration possible, and the members of the 2008 and 2009 Chinese expedition teams for their efforts installing and servicing PLATO. We acknowledge funding from the Chinese PANDA International Polar Year project, the Chinese Academy of Science, the National Natural Science Foundation of China, the US National Science Foundation, the Australian Antarctic Division, and the Australian Research Council through the Discovery Projects and Linkage International schemes.

\section{References}

Ashley, M. C. B., Burton, M. G., Lawrence, J. S., \& Storey, J. W. V. 2004, Astron. Nachr, No. 6-8, 619-625

Bonner, C. S., Ashley, M. C. B., Lawrence, J. S., Luong-Van, D. M., \& Storey, J. W. V. 2009, Acoustics Australia, 37, 47-51

Hengst, S., Luong-Van, D. M., Everett, J. R., Lawrence, J. S., Ashley, M. C. B., Castel, D., \& Storey, J. W. V., 2009, Int. J.Energy Res., DOI: 10.1002/er.1595, in press

Lawrence, J. S., Ashley, M. C. B., Tokovinin, A., \& Travouillon, T. 2004, Nature, 431, 278-281

Lawrence, J. S., Ashley, M. C. B., Hengst, S., Luong-Van, D. M., Storey, J. W. V., Yang, H., Zhou, X., \& Zhu, Z. 2009, Rev. Sci. Inst., 80, 064501-1-064501-10

Yang, H. et al. 2009, PASP, 121, 174-184 